\title{
Metal enrichment processes in the intra-cluster medium
}

\author{
S. Schindler ${ }^{1}$, W. Kapferer ${ }^{1}$, W. Domainko ${ }^{1}$, M. Mair ${ }^{1}$, E. van Kampen ${ }^{1}$, T. Kronberger ${ }^{1}$, S. Kimeswenger ${ }^{1}$, \\ M. Ruffert ${ }^{2}$, O. Mangete ${ }^{2}$, and D. Breitschwerdt ${ }^{3}$ \\ ${ }^{1}$ Institut für Astrophysik, Leopold-Franzens Universität Innsbruck, Technikerstraße 25, 6020 Innsbruck, Austria \\ http://astro.uibk.ac.at/astroneu/hydroskiteam/index.htm \\ e-mail: sabine.schindler@uibk.ac.at \\ 2 School of Mathematics, University of Edinburgh, Edinburgh EH9 3JZ, Scotland, UK \\ 3 Institut für Astronomie, Universität Wien, Türkenschanzstr. 17, 1180 Vienna, Austria
}

Received 11 February 2005 / Accepted 6 April 2005

\begin{abstract}
We present numerical simulations of galaxy clusters which include interaction processes between the galaxies and the intra-cluster gas. The considered interaction processes are galactic winds and ram-pressure stripping, which both transfer metal-enriched interstellar medium into the intra-cluster gas and hence increase its metallicity. We investigate the efficiency and time evolution of the interaction processes by simulated metallicity maps, which are directly comparable to those obtained from X-ray observations. We find that ram-pressure stripping is more efficient than quiet (i.e. non-starburst driven) galactic winds in the redshift interval between 1 and 0 . The expelled metals are not mixed immediately with the intra-cluster gas, but inhomogeneities are visible in the metallicity maps. Even stripes of higher metallicity that a single galaxy has left behind can be seen. The spatial distribution of the metals transported by ram-pressure stripping and by galactic winds are very different for massive clusters: the former process yields a centrally concentrated metal distribution while the latter results in an extended metal distribution.
\end{abstract}

Key words. galaxies: clusters: general - galaxies: abundances - galaxies: interactions - galaxies: ISM -

$\mathrm{X}$-ray: galaxies: clusters

\section{Introduction}

The components of clusters of galaxies - galaxies and intracluster medium (ICM) - interact with each other in many ways. There is more and more observational evidence that various types of processes are at work, which remove interstellar medium (ISM) from the galaxies. This metal enriched ISM mixes with the ICM, so that the currently observed ICM is a mixture of both. Hence the metals are a good tracer for the present and past interaction processes between galaxies and ICM. The new X-ray observations by CHANDRA and XMM make it possible to measure not only profiles but 2D distributions of metals: metallicity maps (e.g. Schmidt et al. 2002; Furusho et al. 2003; Sanders et al. 2004; Fukazawa et al. 2004; Hayakawa et al. 2004) showing that the metallicity has clearly a non-uniform, non-spherical distribution.

Already many years ago supernova-driven galactic winds were suggested as a possible ISM transfer mechanism (De Young 1978). Recent observations and simulations indicate that other processes can contribute considerably, as well (e.g. Hayakawa et al. 2004). One process gaining increasing attention is ram-pressure stripping (Gunn \& Gott 1972). In the Virgo cluster alone at least 7 ram-pressure affected spiral galaxies have been found (e.g. Cayatte et al. 1990; Vollmer et al. 2004). Also other processes like galaxy-galaxy interactions
(Kapferer et al. 2005), jets from AGNs and intra-cluster supernovae (Domainko et al. 2004) can contribute to the metal enrichment.

Currently, several approaches are being made to explain the overall enrichment of the ICM by taking into account galactic winds. De Lucia et al. (2004) and Nagashima et al. (2005) use a combination of semi-analytic techniques and $\mathrm{N}$-body simulations to calculate the overall ICM metallicity, but they do not predict the distribution of metals in a cluster. They find that mainly the massive galaxies contribute to the enrichment and that there is a mild metal evolution since $z=1$. Tornatore et al. (2004) do simulations with smoothed particle hydrodynamics that include detailed yields from type Ia and II supernovae, but do not distinguish between the different transport processes. They put most of their emphasis on the amount of iron produced and on metallicity profiles.

Other groups have calculated the effect of ram-pressure stripping on single galaxies (e.g. Abadi et al. 1999; Quilis et al. 2000; Toniazzo \& Schindler 2001; Roediger \& Hensler 2005), but not the effect on the ICM. We take a complementary approach by investigating the transport of the metals from the galaxies into the intra-cluster medium and study in detail the efficiency of the various transport processes. In our simulations presented here, we have taken into account two different transport processes - ram-pressure stripping and galactic winds. 


\section{Simulations}

\subsection{Numerical method}

We use $N$-body and hydrodynamic techniques together with a semi-numerical phenomenological galaxy formation code and various prescriptions for interactions between galaxies and the intra-cluster medium. The core of the programme is a hydrodynamic code with shock capturing scheme (PPM, Colella \& Woodward 1984), multiple grid refinement (Ruffert 1992) and radiative cooling. This hydrodynamic code is combined with an $N$-body tree code (Barnes \& Hut 1986) providing actual galaxy orbits - with constrained random fields as inital conditions (Hoffman \& Ribak 1991) as implemented by van de Weygaert \& Bertschinger (1996). It also includes an improved version of the galaxy formation code by van Kampen et al. (1999), which provides galaxy properties. We use a $\Lambda$ CDM cosmology $\left(\Omega_{\Lambda}=0.7, \Omega_{\mathrm{m}}=0.3, \sigma_{8}=0.93\right.$, and $h=0.7$ ) with $64^{3}$ particles. While the $N$-body code and the galaxy formation trace the whole evolution from the beginning the hydrodynamic covers the redshift interval between 1 and 0 with time steps of about 15 Myrs starting with the initial condition of hydrostatic equilibrium. The hydrodynamic simulation is calculated on 4 levels of nested grids centred at the cluster centre, the largest being $(20 \mathrm{Mpc})^{3}$ and the smallest $(2.5 \mathrm{Mpc})^{3}$. The number of grid cells at each level is $128^{3}$. In addition we use various prescriptions for the different interaction processes which transport metal enriched gas from the galaxies into the ICM. These transport processes are calculated in the full simulation volume.

\subsection{Galactic winds}

The amount of matter ejected by galactic winds is calculated with an approach initiated by Breitschwerdt et al. (1991). The algorithm includes thermal and cosmic ray driven winds in disk galaxies. The wind code requires several galaxy parameters like halo mass, disk mass, spin parameter, scale length of the components, temperature distribution of the ISM, magnetic field strength and gas density distributions as well as stellar density distribution. We perform parameter studies and summarise their results in a look up table for about 1000 different disk galaxies. The database includes galaxies with halo masses of $1 \times 10^{10} M_{\odot}-1 \times 10^{12} M_{\odot}$. In addition 10 different disk masses for a given halo mass (0.01-0.5 $\times$ halo mass) and 10 different spin parameters for each halo-disk combination are calculated. As we want to investigate the contribution of galactic winds to the enrichment of the ICM of quiet, non-starburst disk galaxies (=steady-state winds) the parameters of the ISM and the star formation rate $\left(1 M_{\odot} / \mathrm{yr}\right)$ are kept constant. Following this approach we find an average mass loss rate of about $0.3 M_{\odot} / \mathrm{yr}$. In case of a mass loss due to a galactic winds the ISM is transferred with the corresponding metallicity and a temperature of $5 \times 10^{6} \mathrm{~K}$ into the hydrodynamic simulation at the position of the galaxy.

\subsection{Ram-pressure stripping}

Galaxies in galaxy clusters can suffer mass loss from interaction with the ICM, e.g. ram pressure acts on the gas disk of galaxies moving through the ICM. We assume that gas is stripped off beyond the radius of the galaxy where the restoring gravitational force is equal to the force due to ram-pressure (stripping radius, Gunn \& Gott 1972). We assume for disk galaxies an exponential stellar disk and gas disk. The validity of this approach has been shown by simulations and by comparisons of galaxies in the Virgo cluster affected by ram pressure (Abadi et al. 1999). For supersonic galaxies we follow the Rankine Hugoniot condition to derive the properties of the galaxies surrounding ICM. Mass loss of galaxies moving inclined through the ICM is scaled with the cosine of the inclination angle with respect to the mass loss derived for an uninclined galaxy. Galaxies which are stripped are assumed to have a truncated gas disk and they only lose more gas if increased ram pressure further reduces the stripping radius.

\section{Results}

We present simulations of clusters with different mass: a massive $\left(1.3 \times 10^{15} M_{\odot}\right.$, Cluster 1$)$ and a less massive cluster $(7.4 \times$ $10^{14} M_{\odot}$, Cluster 2). Cluster 2 undergoes a major merger (mass ratio 1:3), whereas Cluster 1 only has small merger events.

In Fig. 1 the X-ray emission weighted metallicity maps are presented for Clusters 1 and 2: only the enrichment that has taken place since redshift 1 has been taken into account. Cluster 1 is shown at redshift $z=0$ with two different enrichment mechanisms, ram-pressure stripping (Panel 1a) and galactic winds (Panel 1b). The two mechanisms result in a very different spatial distribution. Ram-pressure stripping is very efficient in the centre, because there the ICM density is very high $\left(\sim 6 \times 10^{-27} \mathrm{~g} / \mathrm{cm}^{3}\right.$ within a radius of $\left.0.1 \mathrm{Mpc}\right)$ and leads to a centrally concentrated metallicity distribution. On the other hand the high central ICM density yields a high pressure which suppresses galactic winds resulting in a very extended distribution. Apart from the differences in the centre both processes yield pronounced inhomogeneities in the abundances. Single galaxies leave traces of metals in shapes of stripes behind them. Both the centrally concentrated metallicities and the general inhomogeneities are in good agreement with observations. The increase of metallicity (X-ray emission weighted) since redshift 1 averaged over the central cluster region within a radius of $400 \mathrm{kpc}$ is 0.09 and $4 \times 10^{-8}$ in solar units for the rampressure and the winds model, respectively.

The situation looks very different in a less massive cluster. In Cluster 2 the winds are less suppressed. Therefore the winds contribute also to the central metallicity. Inhomogeneities in the abundances do not disperse immediately (compare insets) even though the ICM in this cluster is stirred by a merger. The stripes are gradually spread out and at the end a roughly homogeneous region of high metallicity is present at the centre. A given inhomogeneity at $t=1.7 \mathrm{Gyr}$ will be spread out on average over a 30 times larger volume at the end of the simulation ( $t=8 \mathrm{Gyr}$ ), depending strongly on the local dynamics of the surrounding ICM, e.g. shocks. 


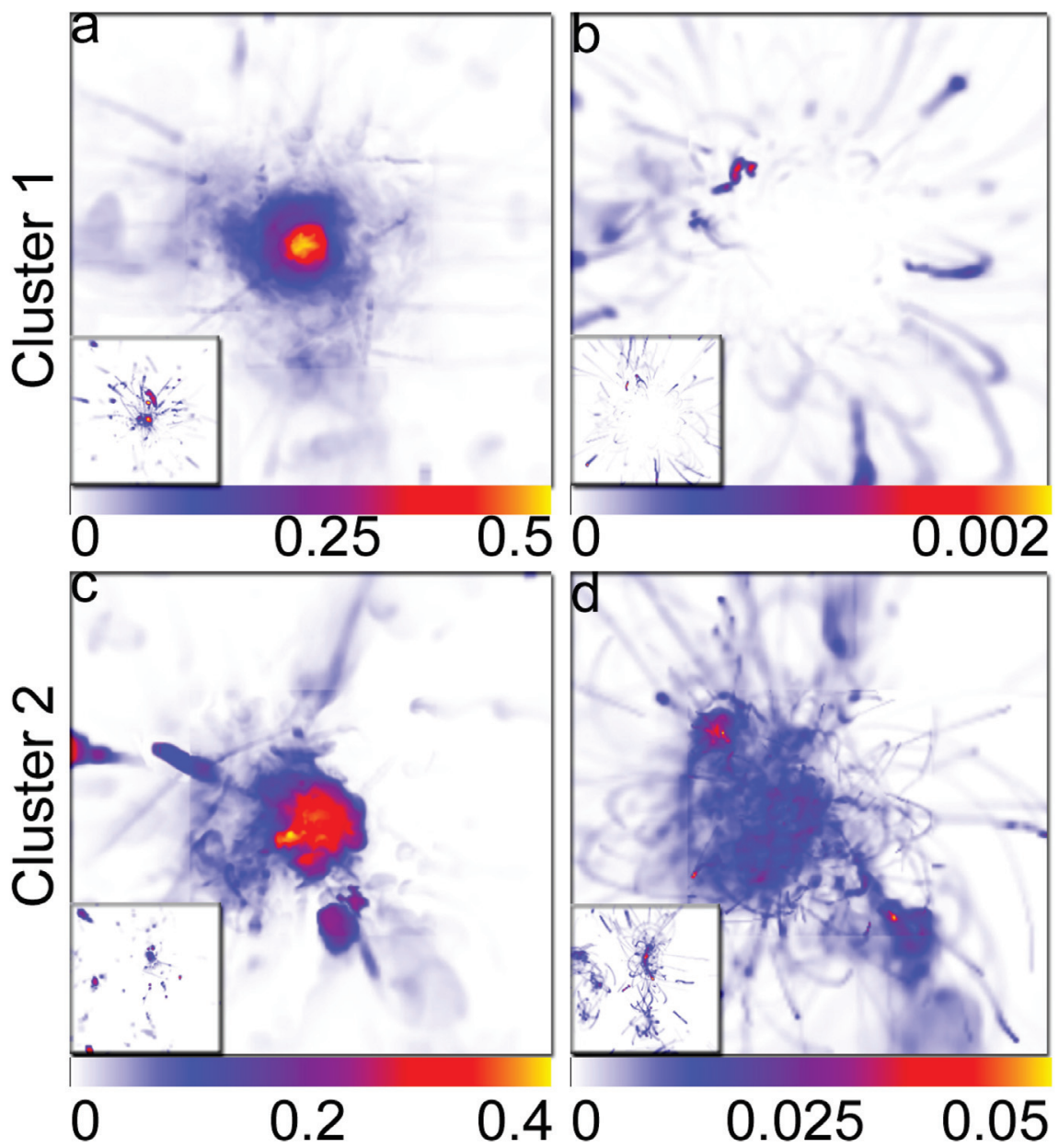

Fig. 1. X-ray weighted metal maps of two simulations at present epoch and insets at an epoch $6.7 \mathrm{Gyr}$ ago (a) and b), Cluster 1: a massive galaxy cluster; c) and d), Cluster 2: a merging cluster). Panels a) and c) show the metal maps for ram-pressure stripping and panels b) and d) those for galactic winds (non-starbursts). The quantities are given in solar abundances and the size of the maps is $5 \mathrm{Mpc}$ on the side. Ram-pressure stripping always yields a centrally concentrated metallicity distribution. The distribution of metals transported by winds depends strongly on the cluster mass: in a massive cluster (b) the winds are suppressed in the centre resulting in a low abundance there. The comparison with the insets shows how the inhomogeneities spread/evolve.

Non-starburst driven winds are less efficient in transporting gas than ram-pressure stripping in the time interval between redshift 1 and 0 investigated here. During the non-merger phases of Cluster 2 ram-pressure stripping is about a factor of 5 more efficient than non-starburst driven winds. Figure 2 shows the evolution of the metal mass loss for ram-pressure stripping and quiet galactic winds in a merger cluster. The different efficiencies are clearly visible. The increase of metallicity (X-ray emission weighted) since redshift 1 averaged over the central cluster region within a radius of $400 \mathrm{kpc}$ is 0.07 and 0.0035 in solar units for the ram-pressure and the winds model, respectively.

The mass loss due to ram-pressure stripping shows large fluctuations compared to galactic winds, because non-starburst driven winds have an almost constant mass loss over a long period of up to $5 \mathrm{Gyr}$, whereas ram-pressure stripping removes huge amounts of gas from a single galaxy on much shorter time scales. During the two merger events (at $3 \mathrm{Gyr}$ and $5.5 \mathrm{Gyr}$ ) the mass loss due to ram pressure is much higher because of the enhanced ICM pressure. In contrast non-starburst driven

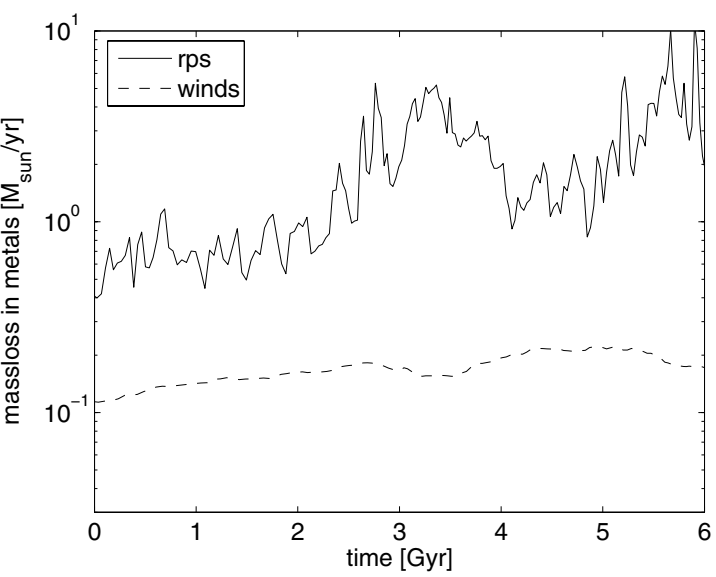

Fig. 2. Evolution of the metal transfer from the ISM to the ICM in solar masses per year for model Cluster 2. The metal mass loss for ram-pressure stripping and for non-starburst driven galactic winds are shown. 
galactic winds are suppressed in galaxies located in the high ICM pressure region. Therefore the mass loss due to winds is slightly lowered during mergers.

\section{Summary and conclusions}

Different enrichment mechanisms - ram-pressure stripping and galactic winds - yield different metallicity distributions.

- In massive clusters ram-pressure stripping provides a much more centrally concentrated distribution than galactic winds, because galactic winds can be suppressed in the cluster centre while ram-pressure stripping is most efficient there.

- Both processes yield abundance distributions with lots of inhomogeneities in good agreement with recent X-ray observations.

- The inhomogeneities are not dispersed immediately, but are gradually spread out.

- The efficiency of gas transfer due to ram-pressure stripping is higher compared to non-starburst driven galactic winds in the redshift interval between 1 and 0 .

The comparison of these results with new detailed observed metallicity maps will yield insights into the various interaction processes between the ICM and the cluster galaxies. The knowledge on their efficiency and timescales will provide information on the formation and evolution of galaxies and their hosting clusters. In the future we will extend the simulations to higher redshifts $(z>1)$ so that we will be able to compare the simulated metallicities directly with the observed metallicity maps. Further simulations including not only more interaction processes, but also distinguishing between different chemical elements are planned.

Acknowledgements. This work was supported by the Austrian Science Foundation FWF (P15868), UniInfrastruktur 2004, Tiroler Wissenschaftsfonds, AUSTRIAN GRID and by a
University of Innsbruck scholarship. Edmund Bertschinger and Rien van de Weygaert are acknowledged for providing their constrained random field code.

\section{References}

Abadi, M. G., Moore, B., \& Bower, R. G. 1999, MNRAS, 308, 947

Barnes, J., \& Hut, P. 1986, Nature, 324, 446

Breitschwerdt, D., Völk, H., \& McKenzie, J. 1991, A\&A, 245, 79

Cayatte, V., van Gorkom, J. H., Balkowski, C., \& Kotanyi, C. 1990, AJ, 100, 604

Colella, P., \& Woodward, P. R. 1984, J. Comp. Phys., 54, 174

De Lucia, G., Kauffmann, G., \& White, S. D. M. 2004, MNRAS, 349, 1101

De Young, D. S. 1978, ApJ, 223, 47

Domainko, W., Gitti, M., Schindler, S., \& Kapferer, W. 2004, A\&A, 425, L21

Fukazawa, Y., Kawano, N., \& Kawashima, K. 2004, ApJ, 606, L109

Furusho, T., Yamasaki, N. Y., \& Ohashi, T. 2003, ApJ, 596, 181

Gunn, J. E., \& Gott, J. R. III 1972, ApJ, 176, 1

Hayakawa, A., Furusho, T., Yamasaki, N. Y., Ishida, M., \& Ohashi, T. 2004, PASJ, 56, 743

Hoffman, Y., \& Ribak, E. 1991, ApJ, 380, L5

Kapferer, W., Knapp, A., Schindler, S., Kimeswenger, S., \& van Kampen, E. 2005, A\&A, in press

[arXiv: astro-ph/0503559]

Nagashima, M., Lacey, C. G., Baugh, C. M., Frenk, C. S., \& Cole, S. 2005, MNRAS, 358, 1247

Quilis, V., Moore, B., \& Bower, R. 2000, Science, 288, 1617

Roediger, E., \& Hensler, G. 2005, A\&A, 433, 875

Ruffert, M. 1992, A\&A, 265, 82

Sanders, J. S., Fabian, A. C., Allen, S., \& Schmidt, R. W. 2004, MNRAS, 349, 952

Schmidt, R. W., Fabian, A. C., \& Sanders, J. 2002, MNRAS, 337, 71

Toniazzo, T., \& Schindler, S. 2001, MNRAS, 325, 509

Tornatore, L., Borgani, S., Matteucci, F., Recchi, S., \& Tozzi, P. 2004, MNRAS, 349, L19

van de Weygaert, R., \& Bertschinger, E. 1996, MNRAS, 281, 84

van Kampen, E., Jiminez, R., \& Peacock, J 1999, MNRAS, 310, 43

Vollmer, B., Beck, R., Kenney, J. D. P., \& van Gorkom, J. H. 2004, AJ, 127, 3375 